23

\title{
Спектральное распознавание объектов с помощью многооконных акустооптических фильтров
}

\author{
(C) В.Э. Пожар ${ }^{1,2}$, Д.Ю. Великовский ${ }^{1}$ \\ ${ }^{1}$ Научно-технологический центр уникального приборостроения РАН, \\ 117342 Москва, Россия \\ ${ }^{2}$ МГТУ им. Н.Э. Баумана, \\ 105005 Москва, Россия \\ e-mail: vitold@ntcup.ru
}

Поступила в редакцию 03.02.2020 г.

В окончательной редакции 18.03.2020 г.

Принята к публикации 27.03.2020 г.

Рассмотрена задача обнаружения объектов по их спектральным признакам при гиперспектральной съемке. Описаны основные проблемы, препятствующие эффективному и оперативному решению задачи. Обоснован метод дискриминации объектов от фона в реальном времени, основанный на использовании многооконных перестраиваемых спектральных фильтров. Сформулированы принципы работы и технической реализации этих устройств. Обсуждены допущения, лежащие в основе метода, и его особенности. Предложен оригинальный способ реализации на основе модулируемого акустооптического фильтра. Сделан вывод о принципиальной реализуемости рассмотренных методов на основе новой технологии многооконной акустооптической фильтрации, его перспективности, быстродействии, оперативности и гибкости.

Ключевые слова: гиперспектральный анализ, экспресс-анализ, многооконный акустооптический фильтр, модуляция акустооптического фильтра.

DOI: $10.21883 /$ OS.2020.07.49578.107-20

\section{Введение}

В настоящее время с развитием разнообразных подвижных платформ для гиперспектральных (ГС) оптических систем возросла актуальность методов, позволяющих проводить анализ и принимать решения в реальном времени. Такие системы широко используются, в частности, в задачах дистанционного зондирования поверхности Земли и мониторинга отдельных участков. Их применяют на различных платформах: от космических аппаратов, ведущих регулярный мониторинг всей поверхности планеты, до беспилотных летательных аппаратов (БПЛА) и наземных подвижных носителей, используемых для решения частных локальных задач. В последнем случае решаемые задачи могут быстро изменяться непосредственно в ходе одного рейса. Для оперативного принятия решений такие системы должны осуществлять анализ данных непосредственно „на борту“ и в реальном времени.

В ходе гиперспектральной съемки формируется массив спектральных изображений $I(x, y ; \lambda)$, каждое из которых представляет собой снимок подстилающей поверхности (сцены) в узкой полосе длин волн $\lambda(x, y-$ координаты точек изображения). Обработка и анализ такого „гиперкуба“ данных требует значительных вычислительных ресурсов и времени, что трудно реализуемо при базировании ГС систем на мобильной платформе [1-3]. Поэтому интерес представляет разработка специализированных средств и методов регистрации и анализа $[2,4]$, которые позволяли бы выполнять некоторые базовые операции, такие как поиск и обнаружение объектов с известным спектром в реальном времени.

Принципиальный подход к разрешению этой проблемы можно проиллюстрировать следующим простейшим примером. Предположим, что для конкретной решаемой задачи интерес представляют объекты определенного типа, характеризующиеся известным спектральным образом, распределенные неизвестным образом по наблюдаемому участку поверхности (сцены). Будем считать, что число различных элементов сцены ограничено, а их спектры известны. В такой формулировке задача заключается в обнаружении объекта на различном фоне, причем спектры объекта и фонов известны, а их пространственное положение - нет. Поскольку получение информации о фоне в задаче не требуется, то решение задачи заключается в построении системы, не чувствительной к фону, но реагирующей на спектр объекта. В этом случае система способна обнаружить и объект, размеры которого на фотоприемной матрице меньше размера пикселя [4]. Такая спектральная система может быть в принципе реализована на основе быстро перестраиваемых акустооптических (AО) фильтров с программно синтезируемой передаточной характеристикой [3-5].

Ниже рассмотрены: устройство многооконных $\mathrm{AO}$ фильтров, являющихся основой системы; задачи, сводящиеся к описанной упрощенной модели объекта; математическая основа решения задачи и техническая 

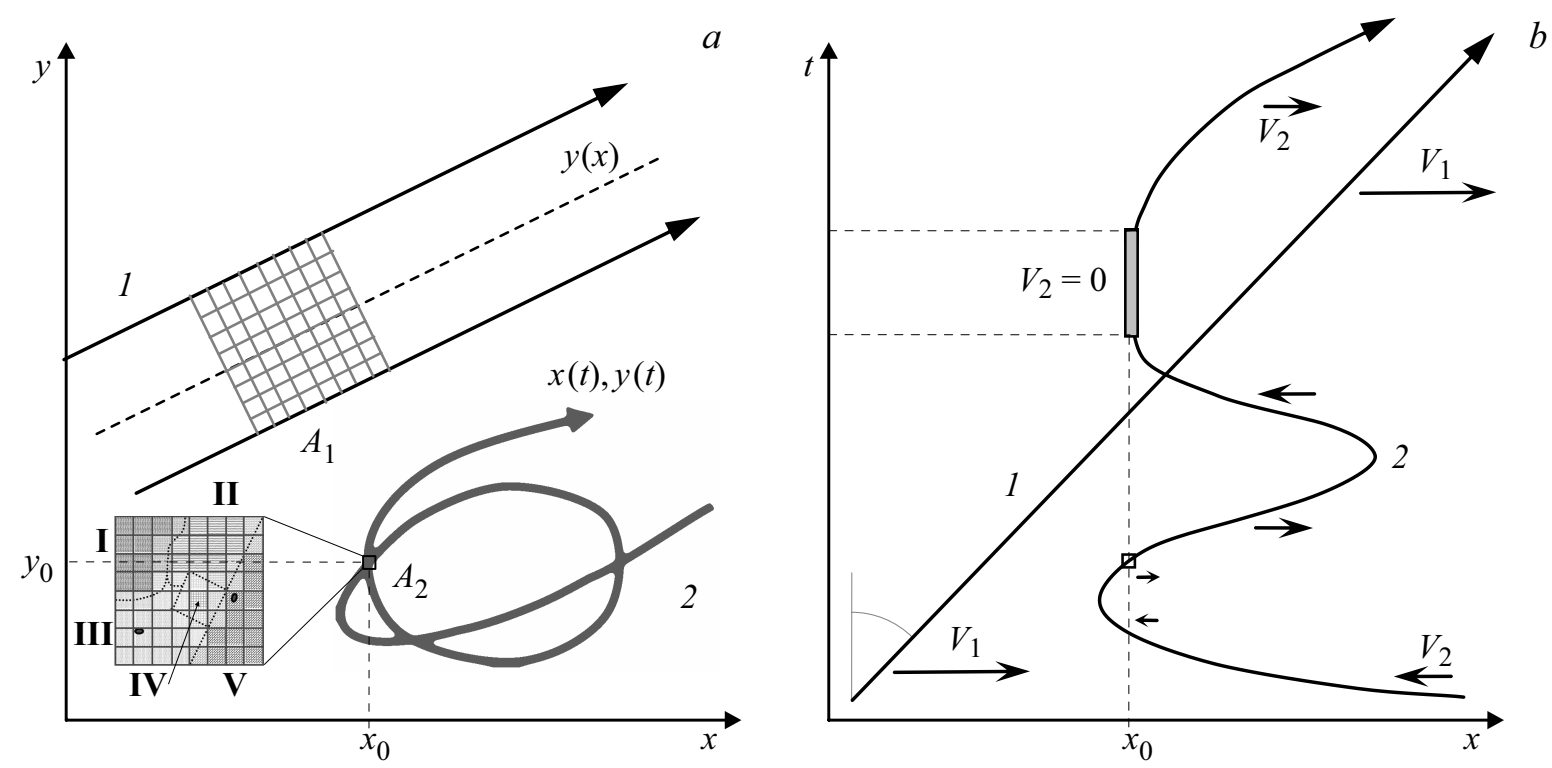

Рис. 1. Особенности процедуры мониторинга при размещении ГС системы на борту спутника (1) и на БПЛА (2): (a) формы траекторий движения и поля зрения $A$ систем, на врезке - для примера приведена локальная карта поверхности с пятью (I-V) типами фона и двумя экземплярами объекта (мелкие черные овалы); $(b)$ временные графики $x(t)$ движения платформ (стрелками обозначена скорость движения $V$, отмечен также интервал зависания ГС системы над объектом при втором проходе).

реализация системы. Отдельно обсуждается новая идея реализации таких систем на основе АO фильтра в режиме модуляции его функции пропускания. И поскольку обсуждаемая общая задача обнаружения для наглядности ниже рассмотрена на примере задачи мониторинга поверхности, предварительно описаны возможности, которые открывает размещение управляемых гиперспектральных систем на платформе БПЛА.

\section{Особенности размещения гиперспектрометров на БПЛА}

С целью выработки требований к ГС системам для управляемых платформ (например, БПЛА) следует сравнить особенности их размещения на этой платформе в сравнении с мониторингом с борта космических аппаратов, данные которого после обработки широко используются на практике. На рис. 1 проиллюстрированы основные отличия методов регистрации ГС данных в случае использования двух сравниваемых платформ.

Как видно, в отличие от спутниковых приборов, которые движутся циклическим образом по заданной орбите, проекция которой локально представляет собой прямую линию, ГС система, располагаемая на БПЛА, во-первых, может двигаться по любой заданной траектории, вовторых, может осуществлять мониторинг оперативно в любое время дня. Кроме того, в случае использования платформы на основе геликоптеров (мультикоптеров) появляются дополнительные возможности: возвратиться в точку обнаружения объекта $\left(x_{0}, y_{0}\right)$; осуществлять зависание в исследуемом месте, обеспечивая достаточно долгое накопление сигнала или непрерывное отслеживание состояния объекта; регулировать высоту наблюдения, а соответственно масштаб и степень детализации изображений.

Главные особенности сравниваемых методов регистрации сведены в таблице. Одним из наиболее важных отличий является то, что спутниковая ГС система выполняет сканирование поверхности Земли с довольно большим периодом как по пространству, так и по времени, тогда как ГС система на управляемой мобильной платформе позволяет реализовать локальный в пространстве и времени, гибкий, управляемый режим мониторинга, который, в частности, дает возможность изменять характеристики регистрации (траекторию, высоту, время накопления и др.) в соответствии с получаемой информацией, т. е. адаптивно [2].

Таким образом, возникает необходимость создания ГС системы анализа с характеристиками, адекватными возможностям таких платформ, как БПЛА. Такая система может быть построена на основе АО перестраиваемых фильтров [6-8], которые обладают способностью быстро $(1-10 \mu \mathrm{s})$ скачкообразно перестраиваться по спектру с одной длины волны на другую, задаваемую произвольно, что позволяет гибко варьировать алгоритм анализа. Кроме того, они компактны, устойчивы к различным условиям эксплуатации, а в схеме двойной фильтрации позволяют минимизировать искажения изображения. Спектральные системы на основе АО фильтров дают возможность регистрировать серию изображений $I\left(x, y ; \lambda_{i}\right)$ на произвольных длинах волн $\lambda_{i}$. Эти фильтры используются в методах спектральной визуализации структуры объектов (spectral imaging). 

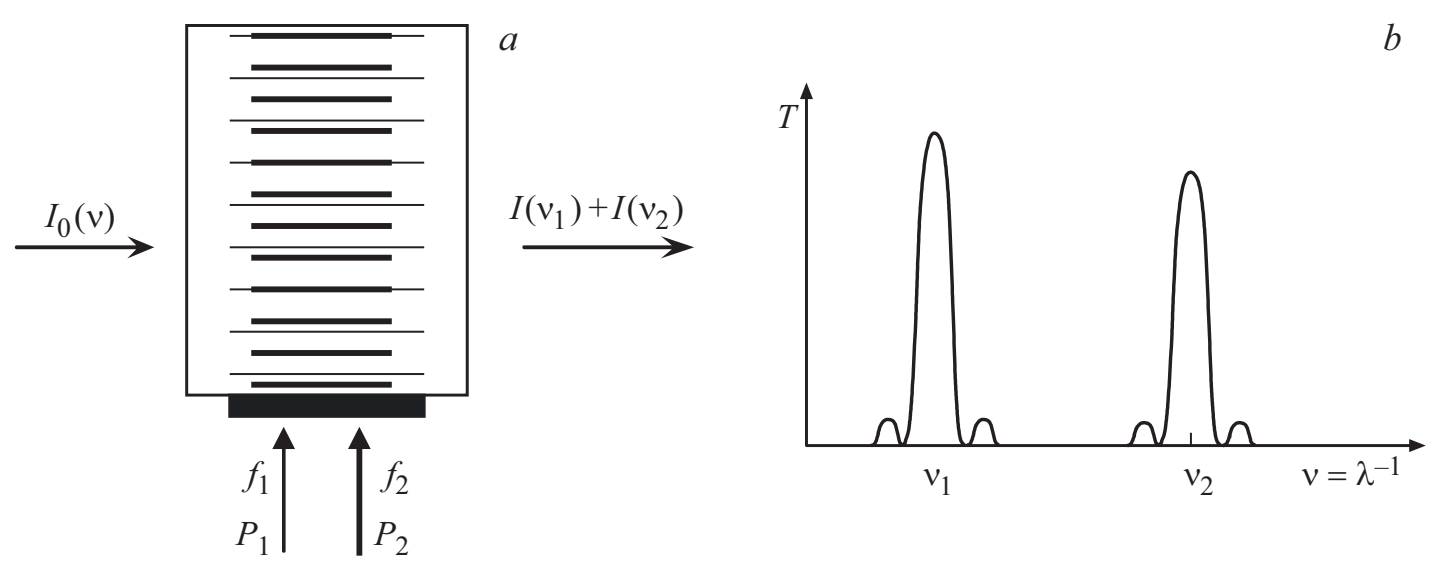

Pис. 2. Многооконный АО фильтр: (a) принцип работы; $(b)$ функция пропускания. $I_{0}(v)$ - спектр интенсивности анализируемого светового потока; $I\left(v_{1}\right), I\left(v_{2}\right)$ - интенсивности выделенных спектральных компонент на соответствующих оптических частотах; $f_{1}, f_{2}, P_{1}, P_{2}$ - частоты и мощности возбуждаемых акустических волн, $T(v)=I(v) / I_{0}(v)$ - спектральный коэффициент пропускания.

Сравнение мониторинга с разных платформ

\begin{tabular}{c|c|c|c|c|c}
\hline Носитель & Траектория & $\begin{array}{c}\text { Высота } \\
\text { наблюдения }\end{array}$ & $\begin{array}{c}\text { Временной } \\
\text { доступ }\end{array}$ & $\begin{array}{c}\text { Временной } \\
\text { интервал }\end{array}$ & $\begin{array}{c}\text { Режим } \\
\text { мониторинга }\end{array}$ \\
\hline Спутник & фиксированная & орбита & периодический & фиксированный & сканирование \\
\hline БПЛА & произвольная & регулируемая & произвольный & ограниченный & управляемый
\end{tabular}

Для оптических систем с указанными свойствами в настоящей работе представлен специализированный метод экспресс-анализа, позволяющий решать определенный тип задач, связанный с обнаружением спектральных объектов в реальном времени. Этот метод базируется на использовании многооконных АО фильтров и на принципах корреляционной, дифференциальной, модуляционной спектрометрии.

\section{Многооконный фильтр}

Многооконный АО фильтр $[3,9,10]$ позволяет сформировать ряд полос пропускания путем одновременного возбуждения в кристалле (рис. 2) акустических волн нескольких разных частот $f_{1}, f_{2}, \ldots$, которые за счет упруго-оптического эффекта вызывают брэгговскую дифракцию соответствующих спектральных компонент света $v_{i} \sim f_{i}(v \equiv 1 / \lambda-$ пространственная оптическая частота света). Регулировкой мощности возбуждаемых акустических волн $P_{i}$ можно варьировать коэффициент пропускания каждого окна $T_{i}$.

Среди областей перспективного применения таких фильтров - согласованная фильтрация полихроматических оптических сигналов в линиях связи по стандарту OCDMA (c разделением доступа за счет оптического кодирования) [3], корреляционная абсорбционная спектроскопия [10] и формирование излучения с требуемым спектром [11].

\section{Модель объекта измерения}

Одной из типичных задач мониторинга с борта БПЛА является поиск объектов на фоне других элементов подстилающей поверхности (рис. 3). При этом можно считать, что спектры всех типов объектов и всех фонов известны. Наиболее надежно осуществлять обнаружение объекта с использованием как спектральных, так и пространственных признаков. Однако во многих случаях информацию о форме использовать невозможно (например, при субпиксельном видимом размере объекта). В настоящей работе рассматривается задача поиска элементов изображения сцены по их спектральным признакам. В этом случае необходимо проверить каждый пиксель изображения на наличие в нем этих признаков.

Такая постановка задачи предполагает за один снимок определять наличие объекта только одного типа с данным спектром. Если необходимо обнаружить и другие типы объектов - с другими спектрами, это можно сделать последовательно с соответствующей настройкой функции пропускания системы $T(v)$. С учетом малого времени перестройки АО фильтров $\left(10^{-5} \mathrm{~s}\right)$ и относительно малой скорости полета БПЛА смещение последовательных кадров незначительно.

Данный подход существенно отличается от ранее обсуждавшихся $[4,5,12]$, которые ориентированы на регистрацию со спутников и определение несколько типов объектов сразу. Для этого необходимо производить несколько спектральных снимков на кадр и приходится 


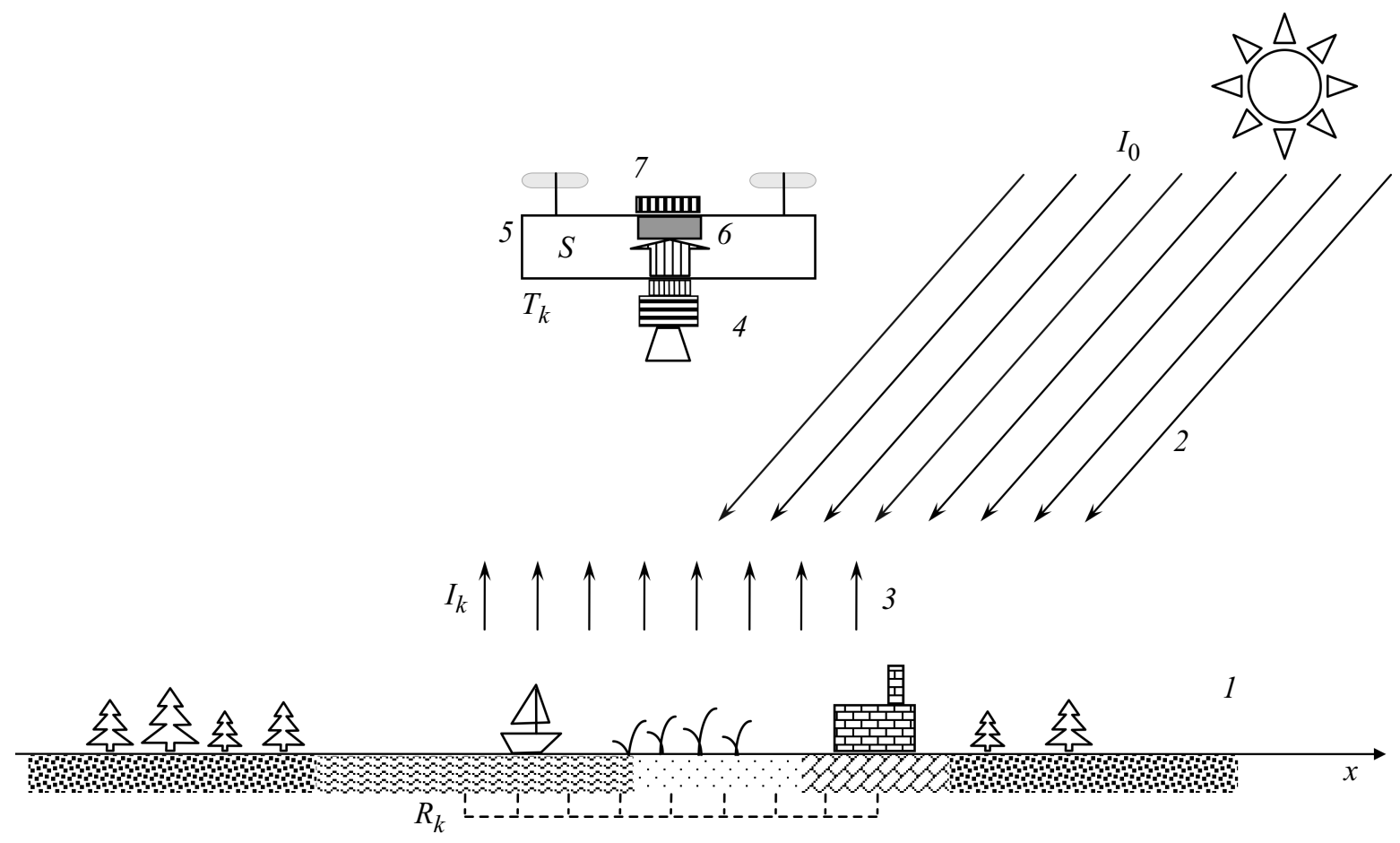

Рис. 3. Схема измерений: 1 - подстилающая поверхность, содержащая разные типы фонов и объекты; 2 - падающий световой поток $I_{0}, 3$ - отраженный световой поток $I_{k}, 4$ - гиперспектрометр (объектив, АО фильтр и матричный фотодетектор), 5 БПЛА, 6 - микропроцессор, 7 - спектроанализатор. Внизу - „поля зрения“ пикселей. $R_{k}(x)-$ коэффициент отражения поверхности в пикселе на длине волны $\lambda_{k}, T_{k}$ - коэффициент пропускания АО фильтра в $k$-й спектральной полосе, $S(x)-$ сигнал фотодетектора от разных элементов поверхности.

делать ряд дополнительных предположений (строгое разделение на объекты и фоны, отсутствие перекрытия фонов, редкое расположение объектов и др.) [4].

В отличие от этого в данном подходе в каждом отдельном измерении (снимке) подстилающей поверхности система настроена на регистрацию отдельной спектральной компоненты из библиотеки образов. Для разрешимости этой задачи общее число спектральных каналов $(K)$ должно превышать число разных спектральных образов $(M): K \geq M$. С учетом того, что число спектральных каналов АО фильтра может составлять $10^{3}-10^{4}$, рассматриваемый подход может в принципе применяться к довольно сложным по спектральному составу задачам.

Считая, что спектр в каждом пикселе $x, y$ складывается из вкладов всех спектральных компонентов в соответствии с их относительным содержанием $b_{m}$ в элементе изображения, соответствующем этому пикселю, можно записать световой поток на длине волны $\lambda_{k}$ в виде

$$
\begin{gathered}
I_{k}(x, y)=I_{0}\left(B_{k 0} b_{0}(x, y)+\sum_{m=1}^{M-1} B_{k m} b_{m}(x, y)\right) \\
\equiv I_{0} R_{k}(x, y),
\end{gathered}
$$

где $I_{0}$ - яркость источника освещения (Солнца), которая для простоты изложения считается однородной по спектру (принципы учета неоднородности обсуждаются ниже), $B_{k m}=B_{m}\left(v_{k}\right), B_{k 0}=B_{0}\left(v_{k}\right)$ - известные спектральные коэффициенты отражения фонов и объекта на оптических частотах $v_{k}(k=1, \ldots, K)$, соответствующих спектральным полосам гиперспектрометра, $b_{m}$, $b_{0}$ - весовые вклады в данном пикселе фонов и объекта, так что $\Sigma b_{m}+b_{0}=1, R_{k}-$ коэффициент отражения на длине волны $k$ в данном пикселе. Поскольку число уравнений $K$ превышает число неизвестных $b_{m}, b_{0}$, одиночное измерение спектра позволяет найти приближенное решение системы (1), погрешность которого определяется отношением сигнал-шум.

Этот классический подход к решению спектроскопической задачи требует использования быстродействующего изображающего спектрометра, что делает его труднореализуемым, особенно во внелабораторных условиях. Следует добавить, что ввиду схожести спектра у многих фонов система уравнений может оказаться плохо определена, а ее полное решение может оказаться неустойчивым к шумам и иным случайным помехам. Все это, однако, не влияет напрямую на решение рассматриваемой упрощенной задачи - выявления наличия (или отсутствия) искомого объекта в каждом анализируемом пикселе изображения, а также определение его относительного вклада $b_{0}$, который может служить оценкой относительной площади, занимаемой объектом. 
Для этой задачи неточность определения вкладов других объектов $b_{m}$ не имеет значения.

Решение поставленной задачи основано на описанном во введении подходе - нахождении такого спектрального фильтра $T\left(v_{k}\right)$, сигнал которого был бы пропорционален $b_{0}$, и его реализации на основе многооконных $\mathrm{AO}$ фильтров.

\section{Метод}

В ходе измерений, выполняемых гиперспектрометром (рис. 3), спектр отражения $R_{k}=I_{k} / I_{0}$ в каждом отдельном пикселе описывается одним числом (фототоком):

$$
S[\mathbf{R}]=\int I(v) T(v) \beta(v) d v=\beta I_{0} \sum_{k=1}^{K} R_{k} T_{k} \propto \mathbf{R T} .
$$

Здесь $\beta-$ спектральная чувствительность фотоприемника, которая для простоты считается постоянной (обсуждение ниже), $T_{k}=T\left(v_{k}\right)$ - спектральная функция пропускания многооконного АО фильтра, а жирным шрифтом обозначены векторы в $K$-мерном „спектральном“ пространстве. Математически результат измерения определяется проекцией вектора $\mathbf{R}$, характеризующего наблюдаемую сцену, на направление вектора $\mathbf{T}$, описывающего фильтр. Сигнал фотоприемника определяется суммой вкладов всех присутствующих в пикселе компонент и, если выбрать вектор Т ортогональным ко всем фонам $\left(\mathbf{B}_{m} \mathbf{T}=0, m \neq 0\right)$, то сигнал окажется пропорционален доле площади искомого объекта $b_{0}$ в этом пикселе:

$$
S=\beta I_{0} \sum_{m=0}^{M-1} b_{m} \mathbf{B}_{m} \mathbf{T}=\beta I_{0} b_{0} \mathbf{B}_{0} \mathbf{T} .
$$

Поскольку размерность пространства спектральных компонент превосходит число спектральных образов $(K>M)$, такой выбор может быть осуществлен (рис. $4, a)$. Для этого достаточно спроецировать вектор объекта $\mathbf{B}_{0}$ на подпространство $\mathbf{B}_{\Sigma}^{\prime}$, ортогональное подпространству $\mathbf{B}_{\Sigma}$, построенному на векторах фона $\mathbf{B}_{m}$.

Формула (3) показывает, что для вычисления величины $b_{0}$, характеризующей наличие и долю объекта в пикселе, необходимо знать яркость источника света $I_{0}$. Таким образом, при натурных измерениях необходимо измерять яркость Солнца, а в общем случае спектр $I_{0}(v)$, который зависит от высоты солнца, состояния атмосферы, облачности. Учесть спектральную неоднородность источника света, так же как и фотодетектора $\beta(v)$, можно соответствующей „нормировкой“ спектральных образов:

$$
\widetilde{B}_{m}(v)=B_{m}(v) \beta(v) I_{0}(v) /\left(\beta_{\max } I_{\max }\right) .
$$

То есть для периодического контроля спектра Солнца достаточно дополнить систему простейшим оптическим спектроанализатором, например, на дифракционной решетке с параллельной регистрацией спектра на линейку фотоприемников.

\section{Техническая реализация}

Прямая техническая реализация найденной оптимальной функции затруднительна, так как функции $B_{m}(v)-$ неотрицательные, а потому ортогональная им функция $T(v)$ должна быть знакопеременной. В предшествующих работах $[3,5]$ было предложено использовать для измерений двухканальную регистрацию, которая предполагает использование взаимодополняющей пары многооконных АО фильтров и вычитание фотодетектированных сигналов двух этих каналов (рис. 4,b,c). Особенность этой пары АО фильтров заключается в том, что фильтрация в любой спектральной полосе осуществляется только в одном из каналов $\left(T_{+}\right.$или $\left.T_{-}\right)$.

Для примера на рис. 4, $b$ приведены элементы многооконной функции, не чувствительные к однородному фону 1 и к равномерному наклону спектра 4, и более сложные элементы, чувствительные к отдельным пикам и провалам 2 и к непостоянной кривизне функции 3. Таким образом, можно реализовать функцию, соответствующую любому найденному оптимальному вектору $\mathbf{T}$.

Однако знакопеременные функции передачи можно получить и другим способом - при модуляции решетки АО фильтра и фазочувствительной регистрации. В этом случае амплитуда модулированного выходного сигнала описывает значение коэффициента передачи, а фаза сдвиг относительно модулирующего сигнала. Этот режим был уже теоретически обоснован $[13,14]$ и экспериментально реализован $[14,15]$ в виде дифференциального АО спектрометра. Такой спектрометр позволяет выделять различные составляющие выходного фотосигнала: усредненную $\langle S\rangle$, на частоте модуляции $\omega_{\bmod }$, на удвоенной частоте $S\left(2 \omega_{\mathrm{mod}}\right)$. Для режима периодической фазовой манипуляции, когда в течение периода фаза управляющего ультразвукового сигнала скачком меняется на определенную величину $\Delta \varphi$, а затем скачком возвращается к прежнему значению, функции передачи в этих каналах на выходе демодулятора $H_{0}, H_{1}, H_{2}$ имеют вид одиночного окна, его производной и второй производной соответственно (рис. $4, b$ ). Как видно, они имеют вид, подобный элементам, изображенным выше и формируемым двумя многооконными АО фильтрами. Следовательно, эти функции могут быть использованы для построения оптимального многооконного АО фильтра. В этом случае спектрометр имеет лишь один АО фильтр с единственным входом и несколькими выходами.

\section{Заключение}

Представленный метод показывает возможность получать информацию, достаточную для целей обнаружения объекта без решения задачи полного распознавания элементов сцены, чрезвычайно сложной, ресурсоемкой 


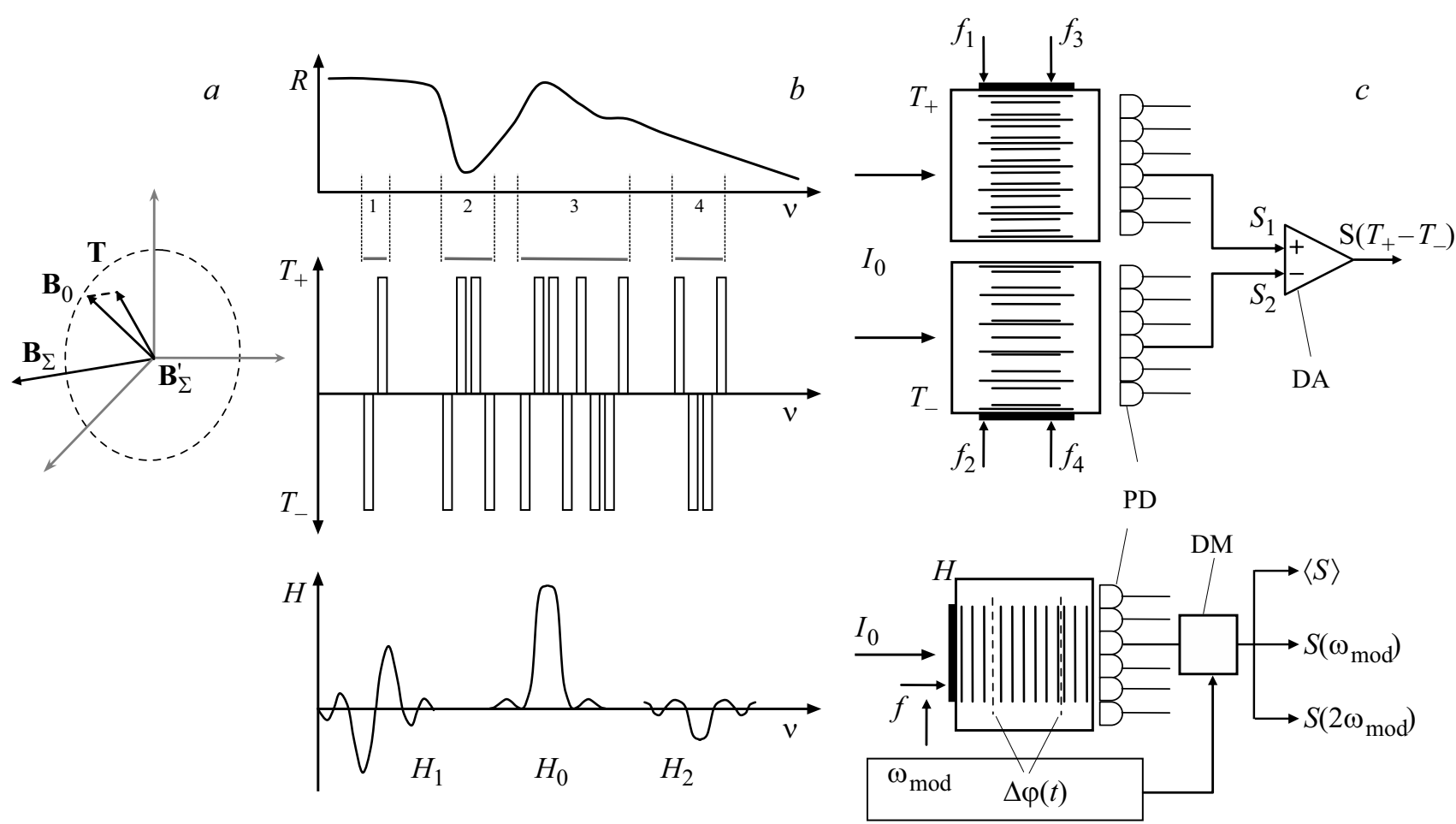

Рис. 4. Техническая реализация оптимального фильтра: $a$ - выбор оптимального вектора T, определяющего форму функции пропускания МО АО фильтра $T(v) ; b$ - спектральные функции коэффициента отражения $R$ (пример), двухканального спектрометра на основе МО АО фильтров $T_{+}, T_{-}$, дифференциального АО спектрометра $H$; $c$ - принципиальная схема двухканального спектрометра на основе многооконного АО фильтра (вверху) и дифференциального АО спектрометра (внизу). $\mathrm{DA}$ - дифференциальный усилитель, PD - линейка фотодетекторов, DM - демодулятор.

и часто неустойчивой. Метод позволяет получать требуемую информацию в реальном времени - за один кадр (при достаточной яркости сцены).

Метод позволяет получать требуемую информацию аппаратно, а потому не требует специальных вычислительных ресурсов. Если необходимо находить несколько разных объектов, то техническая реализация на основе АО перестраиваемых фильтров позволяет сформировать требуемую функцию передачи АО фильтра за микросекундные времена. Другим вариантом является одновременное выделение объектов нескольких спектральных типов без их различения.

Для определенности метод проиллюстрирован на примере ГС мониторинга с борта БПЛА. Однако он применим и для других ГС систем, используемых для изучения динамических процессов и быстро меняющихся объектов. В рассмотренном примере используются спектры отражения, но в принципе метод применим и для эмиссионной, флуоресцентной, абсорбционной спектроскопии.

Метод основан на спектральном выявлении объекта, что позволяет выявлять объекты, видимый размер которых меньше пикселя изображения. При обнаружении спектральных признаков объекта высота наблюдения может быть снижена, что позволит увеличить визуаль- ный размер объекта и использовать информацию о его форме.

На точность определения влияет наличие шума, связанное со степенью освещенности сцены, временем накопления сигнала, вибрацией платформы. Метод основан на спектрах различных компонентов сцены, а потому качество и надежность обнаружения основаны на надежности спектральных данных, их полноте и адекватности модели. Если спектры фонов или объектов похожи, их не удастся различить и иными оптическими спектральными методами, т. е. данный метод не сужает круг разрешимых задач. Степень обусловленности может существенно варьироваться в зависимости от вида объектов и фонов: от низкой в задачах спутникового картирования природных ландшафтов (типов почв, растительности) [4] до высокой в задачах абсорбционного газоанализа [16], что в этих примерах связано с типом спектров - гладких и содержащих узкие линии соответственно. Следует отметить, что те же задачи картирования, обнаружения, классификации при наблюдении с высоты полета БПЛА (до $100 \mathrm{~m}$ ) могут иметь более высокую степень обусловленности, а возможность повторных измерений позволяет снизить требования к методу обнаружения ради упрощения системы мониторинга.

Соответственно весьма перспективным представляется создание таких систем на основе одного АО 
фильтра, работающего в режиме модуляции. Для этого необходимо решить задачу синтеза аппаратной функции дифференциального спектрометра, имеющей требуемый знакопеременный профиль. Эта задача требует отдельного теоретического и экспериментального исследования.

\section{Финансирование работы}

Работа выполнена в рамках Госзадания НТЦ УП РАН.

\section{Конфликт интересов}

Авторы заявляют, что у них нет конфликта интересов.

\section{Список литературы}

[1] Пожкар В.Э., Мачихин А.С., Гапонов М.И., Широков С.В., Мазур М.М., Шерышев А.Е. // Светотехника. 2018. № 4. C. 47-50; Pozhar V.E., Machikhin A.S., Gaponov M.I., Shirokov S.V., Mazur M.M., Sheryshev A.E. // Light and Engineering. 2019. V. 27. N 3. P. 99-104.

[2] Gaponov M., Machikhin A., Pozhar V., Shurygin A. // Proc. SPIE. 2017. V. 10466. P. 104661V. doi 10.1117/12.2288303

[3] Проклов В.В., Бышевский-Конопко О.А., Григорьевский В.И. // Радиотехника и электроника. 2013. Т. 58. № 9. C. $905-915$.

[4] Герус А.В., Герус Т.Г. // Физические основы приборостроения. 2015. Т. 4. № 4. С. 70-83.

[5] Shnitser P., Agurok I., Sandomirsky S. // Proc. SPIE. 1997. V. 3159. P. $138-143$. doi org/10.1117/12.284199

[6] Епихин В.М., Кияченко Ю.Ф., Мазур М.М., Мазур Л.И., Пальцев Л.Л., Судденок Ю.А., Шорин В.Н. // Физические основы приборостроения. 2013. Т. 2. № 4. С. 116-125.

[7] Мазур М.М., Судденок Ю.А., Шорин В.Н. // Письма в ЖТФ. 2014. Т. 40. № 4. С. 56-62; Mazur M.M., Suddenok Yu.A., Shorin V.N. // Techn. Phys. Lett. 2014. V. 40. N 2. P. 167.

[8] Мазур М.М., Мазур Л.И., Судденок Ю.А., Шорин В.Н. // Опт. и спектр. 2018. Т. 125. № 4. C. 572-576; Mazur M.M., Mazur L.I., Suddenok Yu.A., Chorin V.N. // Opt. Spectrosc. 2018. V. 125. N 4. P. 272. doi 10.21883/OS.2018.10.46715.164-18

[9] Пожсар В.Э., Пустовойт В.И. // Изв. РАН. Сер. физ. 2015. T. 79. № 10. C. 1375-1380; Pozhar V.E., Pustovoit V.I. // Bull. Russ. Acad. Sci. Physics. 2015. V. 79. N 10. P. 1221. doi $10.7868 / \mathrm{S} 0367676515100245$

[10] Мазур М.М., Судденок Ю.А., Пожсар В.Э. // Опт. и спектр. 2020. Т. 128. В. 2. С. 284-289; Mazur M.M., Suddenok Yu.A., Pozhar V.E. // Opt. Spectrosc. 2020. V. 128. N 2. P. 274-279. doi 10.21883/OS.2020.02.48980.211-19

[11] Machikhin A., Khokhlov D., Pozhar V., Kozlov A., Batshev M., Gorevoy A. // Proc. SPIE. 2018. V. 10815. P. 108150 L. doi $10.1117 / 12.2502756$

[12] Proklov V.V., Byshevski-Konopko O.A., Lugovskoi A.V. // Acta Phys. Polon. A. 2015. V. 127. N 1. P. 29-35. doi 10.12693/APhysPolA.127.29

[13] Пустовойт В.И., Пожсар В.Э. // Радиотехника и электроника. 1998. Т. 43. В. 1. С. 121-127; Pustovoit V.I., Pozhar V.E. // J. Commun. Technol. Electron. 1998. V. 43. N 1. P. $115-120$.
[14] Pozhar V.E., Boritko S.V., Karandin A.V. // Proc. SPIE. 2019. V. 11028. P. 1102825. doi 10.1117/12.2521010

[15] Pozhar V.E., Pustovoit V.I. // Proc. SPIE. 2005. V. 5806. P. 869-873. doi 10.1117/12.623173

[16] Fadeyev A.V., Pozhar V.E. // Proc. SPIE. 2012. V. 8535. P. 85350 C doi $10.1117 / 12.974426$ 